\title{
Time to get on the stress-only bandwagon?
}

\author{
Matthew W. Parker, MD $\mathbb{D}$, ${ }^{\mathrm{a}}$ and Gary V. Heller, MD, PhD ${ }^{\mathrm{b}}$ \\ a Division of Cardiovascular Medicine, University of Massachusetts Medical Center, Worcester, \\ MA \\ b Gagnon Cardiovascular Institute, Morristown Medical Center, Morristown, NJ
}

Received Sep 2, 2016; accepted Sep 6, 2016

doi:10.1007/s12350-016-0691-0

\section{See related article, pp. 809-816}

Growing concerns for SPECT imaging relate to its overall radiation exposure to patients as well as laboratory inefficiency. A means of reducing both radiation exposure and protocol time is to perform "stress-only" imaging in selected patients. This involves performing either exercise or pharmacologic stress with myocardial perfusion imaging and then deciding whether to perform rest imaging based on the stress findings. Conceptually, this can significantly reduce radiation exposure by omitting the resting dose of radiotracer: A $30 \mathrm{mCi}$ stress-only protocol results in a $27 \%$ reduction in patient radiation exposure because the $10 \mathrm{mCi}$ resting dose is omitted entirely and a $10 \mathrm{mCi}$ stress-only protocol represents a $76 \%$ reduction in exposure when the $30 \mathrm{mCi}$ same-day resting dose is omitted (Figure 1). Additionally, total test time is reduced by half in many patients and cost reduced because fewer consumables are used. It has been estimated that with proper patient selection, the percentage of rest studies necessary is as low as $9 \%$. $^{2,3}$ This low percentage is achieved with line-source attenuation correction. Laboratories performing stress-only imaging without some measure of addressing attenuation artifact may experience the necessity for resting studies far higher, as much as $40-60 \% .^{3}$

The strategy of stress-only imaging makes perfect sense particularly as recent data have demonstrated that only $8-10 \%$ of rest/stress studies are abnormal. ${ }^{4}$ Stressonly imaging has been strongly supported by data showing very low event rates following normal stress-

Reprint requests: Gary V. Heller, MD, PhD, Gagnon Cardiovascular institute, Morristown Medical Center, Morristown NJ; garyvheller@gmail.com

J Nucl Cardiol 2018;25:817-9.

$1071-3581 / \$ 34.00$

Copyright (C) 2016 American Society of Nuclear Cardiology. only studies, ${ }^{2,5-13}$ editorials, ${ }^{14,15}$ and recommendations from the recently published ASNC Information Statement. ${ }^{16}$ Despite the data and ASNC recommendations, only a minority of nuclear cardiology laboratories have incorporated stress-only protocols, estimated to be fewer than $1 \%$ of Intersocietal Accreditation Commission (IAC) accredited laboratories. ${ }^{17}$ The high incidence of abnormalities related to attenuation artifact, which requires either attenuation correction equipment or comparison to a resting imaging to diagnose, appears to be a major obstacle to wider use of stress-only imaging, as well as logistics of payment by either private insurers or Medicare. Prone imaging, collecting a separate image (usually in $1 / 2$ the standard acquisition time ${ }^{18}$ ) with the patient lying prone instead of supine, may accomplish similar results, but does not require additional hardware or software. As with dedicated attenuation correction hardware systems, prone imaging has been shown to increase the specificity of stress MPI without sacrificing sensitivity. ${ }^{19}$ While the data for stress-only imaging with attenuation correction is rich, little information is available using prone imaging to adjudicate attenuation artifact for this purpose.

The current report by Gutstein et al. ${ }^{20}$ describes the all-cause mortality and myocardial infarction (MI) rates following stress-only myocardial perfusion with prone imaging for attenuation correction in a real-world setting. The reported protocol was that of performing stress imaging on patients who have no history of MI or heart failure. The decision for further imaging was made using the following criteria: (1) rest injection and imaging, if summed stress score (SSS) greater than or equal to 4 or abnormal LVEF or volume; (2) re-imaging in the prone position, if perfusion was equivocal or mildly abnormal, that is, SSS greater than 0 but less than 4); (3) no further imaging for those with normal stress scans, that is, SSS $=0$ with normal LV size and function. The authors then compared the cardiac event rates among those who were imaged with normal supine (only) stress scans, normal prone stress scans, and normal stress-rest scans. 


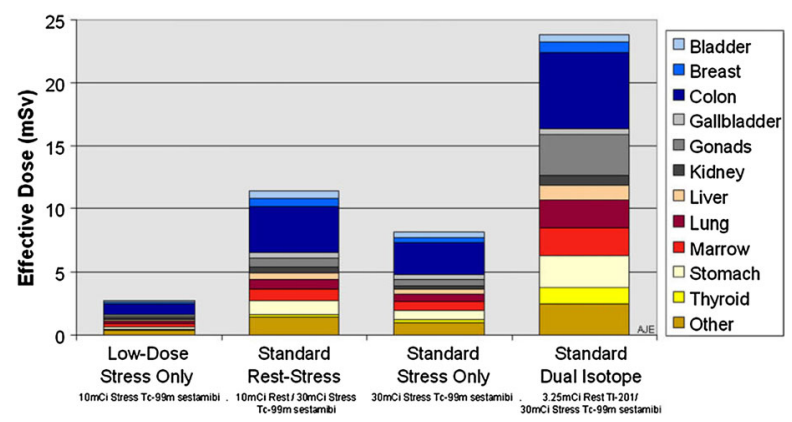

Figure 1. Comparison of effective dose of stress-only protocols in comparison to standard rest-stress protocols. Effective dose from low-dose $(10 \mathrm{mCi}$ Tc-99m) stress-only, standard rest-stress $(10 \mathrm{mCi} \mathrm{Tc}-99 \mathrm{~m} / 30 \mathrm{mCi}$ Tc- $99 \mathrm{~m})$, high-dose stressonly (30 mCi Tc-99m), and standard dual-isotope $(3.24 \mathrm{mCi}$ Tl-201/30 mCi Tc-99m) protocols. $m S v$ millisievert. $m C i$ millicurie. Reproduced from Ref. 1.

They found that those three groups had essentially the same annualized rate of mortality from all causes (1.4\% per year). There was a trend toward more frequent MI during follow-up in patients who ultimately underwent stress and rest imaging. Because the assignment to rest versus prone imaging was not strictly protocol driven and interpretation of the scans was not blinded to clinical data, it is hard to interpret this finding. The combined rate of death from any cause or myocardial infarction was no higher than $2.1 \%$ per year, similar to previous studies of the prognosis of normal prone myocardial perfusion imaging. ${ }^{21,22}$

Prone imaging does not eliminate attenuation artifact but moves attenuating structures to reveal their presence. ${ }^{23}$ A consecutive series of 3234 patients undergoing rest/stress myocardial perfusion imaging in the supine position, the prone position, and supine position with computed tomography-based attenuation correction was reported by Malkerneker et al. ${ }^{24}$ They reported that $18 \%$ of tests were equivocal when prone images were added to the supine images in comparison to $11 \%$ of tests being equivocal when attenuation-corrected images were added to the supine (nonattenuation-corrected) images. ${ }^{24}$ Higher rates of equivocal findings are likely to be amplified when only stress images are available for review and will lead to higher rates of rest imaging. Indeed the percent of rest imaging was substantially higher (34\%) than that observed by Mathur et al. who used line-source attenuation correction (8).

Prone imaging has some downsides in comparison to attenuation correction. Lying prone on the imaging table may be uncomfortable for some patients and may not be feasible in hospitalized patients. Repositioning the patient for a second acquisition may be time consuming, although current ASNC recommendations shorten the acquisition time for this comparison image. The current report suggests that $65 \%$ of patients with equivocal supine stress scans may avoid the radiation exposure associated with a resting scan as well as a separate lengthened protocol by performing a prone image acquisition, so there is clearly a benefit in using prone imaging when dedicated attenuation correction is unavailable.

The retrospective nature of the analysis only addressed those who ultimately had a diagnosis of normal perfusion. An analysis of the subgroups requiring prone, rest, or both in addition to supine stress, as well as the number unable or unwilling to lie prone imaging, would provide a more complete picture of radiation and cost savings when implementing a prone imaging strategy for stress-only imaging and allow the prone strategy to be directly compared to attenuation correction strategies, which have been demonstrated to dramatically reduce equivocal diagnoses and avoid rest imaging in over $91 \%$ of abnormal stress-only scans. ${ }^{2}$

Who should undergo stress-only imaging? In the current report the authors excluded those with prior MI or abnormal ventricular function. The decision may also depend upon logistics. For example, a laboratory with an available reader during working hours may be more liberal in inclusion, while those who read only at the end of the day may be more selective. Generally it is accepted that those with no known CAD as well as those with CAD but no prior infarction are good candidates. It is our feeling based upon practical experience that those with prior coronary artery bypass surgery have a high incidence of abnormal studies and therefore are not good stress-only subjects. Those with heart failure pose other issues such as having reduced ejection fraction at the time of stress, frequently necessitating resting images. Further data on this subset would be useful.

From the perspective of laboratory operations, the retrospective design of the Gutstein paper provides limited direction. LV size and function are often unknown at the onset of testing and so these exclusions are not useful in selecting which patients should undergo a stress-first protocol. Excluding patients with prior myocardial infarction may make sense from a standpoint of studying prognosis, but some of these patients have normal perfusion following treatment of the infarct and therefore may not benefit from exposure to a resting dose of radiation. The large percentage (34\%) of patients who performed prone imaging but still required a resting scan in order to establish a diagnosis of normal perfusion points to this uncertainty in selecting patients. Body mass index, age, or sex may also be important variables and the predictors of a successful stress-only protocol (no rest imaging required) may be different for prone imaging and attenuation correction protocols. We await 
prospective studies to refine these criteria and therefore increase the chances of a successful stress-only protocol.

Gutstein and colleagues have provided a solid starting place for nuclear cardiology laboratories that do not have attenuation correction available interested in radiation reduction and improving efficiency with stress-only imaging. While more data would be important and useful, the current report supports using prone imaging for this purpose. These data hopefully will provide stimulus for many laboratories to begin using stress-only as a means to increase laboratory efficiency and reduce radiation exposure.

\section{References}

1. Henzlova MJ, Duvall WL. The future of SPECT MPI: Time and dose reduction. J Nucl Cardiol 2011;18:580-7. doi:10.1007/ s12350-011-9401-0.

2. Mathur S, Heller GV, Bateman TM, Ruffin R, Yekta A, Katten D, et al. Clinical value of stress-only Tc-99m SPECT imaging: Importance of attenuation correction. J Nucl Cardiol 2013;20:2737. doi:10.1007/s12350-012-9633-7.

3. Gowd BMP, Heller GV, Parker MW. Stress-only SPECT myocardial perfusion imaging: A review. J Nucl Cardiol 2014. doi:10.1007/s12350-014-9944-y.

4. Rozanski A, Gransar H, Hayes SW, Min SW, Friedman J, Thomson $\mathrm{JD}$, et al. Temporal trends in the frequency of inducible myocardial ischemia during cardiac stress testing: 1991 to 2009. J Am Coll Cardiol 2013;61:1054-65. doi:10.1016/j.jacc.2012.11.056.

5. Gal R, Ahmad M. Cost-saving approach to normal technetium99m sestamibi myocardial perfusion scan. Am J Cardiol 1996;78:1047-9.

6. Gibson PB, Demus D, Noto R, Hudson W, Johnson LL. Low event rate for stress-only perfusion imaging in patients evaluated for chest pain. J Am Coll Cardiol 2002;39:999-1004.

7. Chang SM, Nabi F, Xu J, Raza U, Mahmarian JJ. Normal stressonly versus standard stress/rest myocardial perfusion imaging: similar patient mortality with reduced radiation exposure. J Am Coll Cardiol 2010;55:221-30. doi:10.1016/j.jacc.2009.09.022.

8. Duvall WL, Wijetunga MN, Klein TM, Razzouk L, Godbold J, Croft L, et al. The prognosis of a normal stress-only Tc- $99 \mathrm{~m}$ myocardial perfusion imaging study. J Nucl Cardiol 2010;17:3707. doi:10.1007/s12350-010-9210-x.

9. Gemignani AS, Muhlebach SG, Abbott BG, Roye GD, Harrington DT, Arrighi JA. Stress-only or stress/rest myocardial perfusion imaging in patients undergoing evaluation for bariatric surgery. J Nucl Cardiol 2011;18:886-92. doi:10.1007/s12350-011-9405-9.

10. Duvall WL, Wijetunga MN, Klein TM, Hingorani R, Bewley B, Khan S, et al. Stress-only Tc-99m myocardial perfusion imaging in an emergency department chest pain unit. J Emerg Med 2012;42:642-50. doi:10.1016/j.jemermed.2011.05.061.

11. Ueyama T, Takehana K, Maeba H, Iwasaka T. Prognostic value of normal stress-only technetium-99m myocardial perfusion imaging protocol. Comparison with standard stress-rest protocol. Circ J 2012;76:2386-91.

12. Ferreira MJV, Cunha MJ, Albuquerque A, Moreira AP, Ramos D, Costa $\mathrm{G}$, et al. Prognosis of normal stress-only gated-SPECT myocardial perfusion imaging: a single center study. Int J Cardiovasc Imaging 2013;29:1639-44. doi:10.1007/s10554-0130245-3.

13. Edenbrandt L, Ohlsson M, Trägårdh E. Prognosis of patients without perfusion defects with and without rest study in myocardial perfusion scintigraphy. EJNMMI Res 2013;3:58. doi: 10.1186/2191-219X-3-58.

14. Mahmarian JJ. Stress only myocardial perfusion imaging: Is it time for a change? J Nucl Cardiol 2010;17:529-35. doi: 10.1007/s12350-010-9249-8.

15. Bhavnani SP, Heller GV. Stress-only myocardial perfusion imaging ... it is time for a change! J Nucl Cardiol 2011;18:836-9. doi:10.1007/s12350-011-9432-6.

16. Cerqueira MD, Allman KC, Ficaro EP, Hansen CL, Nichols KJ, Thompson RC, et al. Recommendations for reducing radiation exposure in myocardial perfusion imaging. J Nucl Cardiol 2010;17:709-18. doi:10.1007/s12350-010-9244-0.

17. Jerome SD, Tilkemeier PL, Farrell MB, Shaw LJ. Nationwide laboratory adherence to myocardial perfusion imaging radiation dose reduction practices: A report from the intersocietal accreditation commission data repository. JACC Cardiovasc Imaging 2015;8:1170-6. doi:10.1016/j.jcmg.2015.07.008.

18. Nishina H, Slomka PJ, Abidov A, Yoda S, Akincioglu C, Kang X, et al. Combined supine and prone quantitative myocardial perfusion SPECT: method development and clinical validation in patients with no known coronary artery disease. J Nucl Med 2006;47:51-8.

19. Shin JH, Pokharna HK, Williams KA, Mehta R, Ward RP. SPECT myocardial perfusion imaging with prone-only acquisitions: correlation with coronary angiography. J Nucl Cardiol 2009;16:590-6. doi:10.1007/s12350-009-9092-y.

20. Gutstein A, Bental T, Solodky A, Mats I, Zafrir N. Prognosis of stress-only SPECT myocardial perfusion imaging with prone imaging. J Nucl Cardiol. 2016. doi:10.1007/s12350-016-0617-x.

21. Hayes SW, De Lorenzo A, Hachamovitch R, Dhar SC, Hsu P, Cohen I, et al. Prognostic implications of combined prone and supine acquisitions in patients with equivocal or abnormal supine myocardial perfusion SPECT. J Nucl Med 2003;44:1633-40.

22. Worden NE, Lindower PD, Burns TL, Chatterjee K, Weiss RM. A second look with prone SPECT myocardial perfusion imaging reduces the need for angiography in patients at low risk for cardiac death or MI. J Nucl Cardiol 2015;22:115-22. doi:10.1007/s12350014-9934-0.

23. Germano G, Slomka PJ, Berman DS. Supine acceptance of a conventional imaging position may make you less prone to success. J Nucl Cardiol 2010;17:16-8. doi:10.1007/s12350-009-9187-5.

24. Malkerneker D, Brenner R, Martin WH, Sampson UK, Feurer ID, Kronenberg MW, et al. CT-based attenuation correction versus prone imaging to decrease equivocal interpretations of rest/stress Tc-99m tetrofosmin SPECT MPI. J Nucl Cardiol 2007;14:314-23. doi:10.1016/j.nuclcard.2007.02.005. 